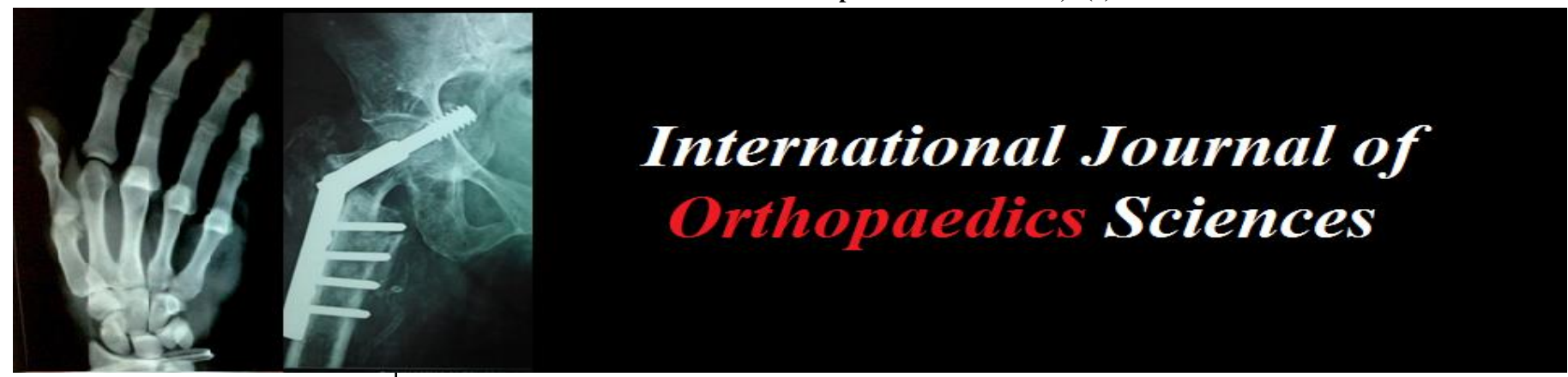

ISSN: $2395-1958$

IJOS 2018; 4(4): 728-731

(C) 2018 IJOS

www.orthopaper.com

Received: 16-08-2018

Accepted: 21-09-2018

Pavankumar Kohli

Professor, Department of

Orthopaedics, Walawalkar Rural

Hospital and Research Centre,

Dervan, Ratnagiri, Maharashtra,

India

Ankush Nawale

Fellow in Spine Surgery,

Walawalkar Rural Hospital and

Research Centre, Dervan,

Ratnagiri, Maharashtra, India

\section{Sushant Chavan}

Fellow in Joint Replacement

Walawalkar Rural Hospital and

Research Centre, Dervan,

Ratnagiri, Maharashtra, India

Meghana Palkhade

Physiotherapist, Walawalkar

Rural Hospital and Research

Centre, Dervan, Ratnagiri,

Maharashtra, India

\section{Sunil Nadkarni}

Professor Trustee, Department of Orthopaedics, Walawalkar Rural Hospital and Research Centre,

Dervan, Ratnagiri, Maharashtra, India

Correspondence

Ankush Nawale

Fellow in Spine Surgery

Walawalkar Rural Hospital and

Research Centre, Dervan,

Ratnagiri, Maharashtra, India

\section{The Dervan protocol: A new improved rehabilitation protocol for faster recovery of knee replacement surgery}

\author{
Pavankumar Kohli, Ankush Nawale, Sushant Chavan, Meghana Palkhade \\ and Sunil Nadkarni
}

DOI: $\underline{\text { https://doi.org/10.22271/ortho.2018.v4.i4i.88 }}$

\section{Abstract}

Introduction: Osteoarthritis is a progressive disorder of joints caused by gradual loss of cartilage most commonly occurring at knee joint in Indian population. Post-operative physiotherapy is one of the most important pre requisite for further recovery.

Traditionally only forward walking is studied, practiced in all rehabilitation protocols. Drawing clues from Yoga and martial arts we have studied the effect of backward walking in post operative rehabilitation of knee replacement patients. Changing basic approach of Rehabilitation has been done for the first time in our knowledge.

Materials \& Methods: This study was done in Walawalkar Rural Hospital and research centre, Dervan, Ratnagiri, Maharashtra. This was a randomized control study.

34 postoperative Patients were randomized into 2 groups. Pre-Operative and Post-Operative assessment was done with VAS score and Modified Oxford Knee Score at the end of 2 weeks.

Results: Backward walking group shows $64.4 \%$ additional improvement in post-operative Modified Oxford knee score over forward walking group. There is significant improvement in pain score (VAS) in backward walking group at the end of 2 weeks.

Discussion: The above results conclude that backward walking reduces significantly the impact force upon contact, foot contact pattern and lower extremity kinematic pattern. Range of Motion at the knee joint is reduced during backward walking incorporating a nearly isometric pattern following contact compared to a more stressful eccentric loading in the traditional forward walk rehab protocol. This can be especially advantageous for rehabilitation of knee joint surgeries along with better proprioception highlighting beneficial effects of backward walking like strengthening of Anti-gravity muscles, Quadriceps loading, Stretching of Post Capsule.

Keywords: Backward walk, reverse walk, knee replacement, rehabilitation, protocol, recovery

\section{Introduction}

Osteoarthritis is a progressive disorder of joints caused by gradual loss of cartilage most commonly occurring at knee joint in Indian population most commonly associated with pain, stiffness, swelling, reduced exercise tolerance, difficulty climbing stairs, increased risk of fall and decreased cadence ${ }^{[1,3,4]}$.

Post-operative physiotherapy is one of the most important aspects for early recovery. There are different protocols used for post-operative physiotherapy worldwide which include Strengthening exercises, proprioception training, gait training etc. ${ }^{[1,2,5,6]}$ However none of them featured on backward walk as primary factor for mobilization.

Reverse walking \& running has been practiced since many years in martial arts, Tai chi \& traditional Yoga techniques. Recent studies have found advantages in increased caloric expenditure, sagittal balance, mindfulness \& state of being.

This is the first time that this protocol has been used in postoperative rehabilitation to our knowledge, with very encouraging results. We recommend its use in the early \& middle phases of rehabilitation from knee surgery.

This study is a Comparative study between forward and backward walking in post-Operative rehabilitation highlighting beneficial effects of backward walking like strengthening of Antigravity muscles, Quadriceps loading, Stretching of Post Capsule and proprioception and mindfulness ${ }^{[7,8]}$ 
Hence this study aims toward establishing new post-operative rehabilitation protocol for faster recovery in knee surgery

\section{Materials and Methods}

This study was done in Walawalkar Rural Hospital and research centre, a tertiary hospital, Dervan, Ratnagiri district, Maharashtra, India. This was a randomized control study. Consent was taken from all patients.

1. Study design: experimental study

2. Inclusion criteria

a) Subjects aged between 40years to 80years, both male and female

b) Subjects who underwent Total Knee Replacement / Unicondylar Knee Replacement who are ambulated

3. Exclusion criteria

a) Any lower limb surgery in past

b) Any active infection

c) Elderly subject with neurological deficit

d) Delayed Rehab due to pain, Effusion, Inflammation

\section{Sample size- 34 subjects}

\section{Methods}

Patients were randomized into 2 groups. Pre-Operative and

Post-operative assessment was done with VAS score $[9,10]$ (Appendix 1) and Modified Oxford Knee Score (Appendix 2). Physiotherapy was started on Post-Operative day 1 as per the patient's tolerance. First group was managed with conventional physiotherapy protocol consisting quadriceps, hip abductor, vastusmedialis, obliques strengthening exercises, proprioception exercises, cycling and forward walking.

Second group B received conventional physiotherapy with the addition of backward walking. Backward walking was included instead for the same amount of time and at the same pace as the forward walking. Patients were made to walk for 10 minutes twice a day. After 2 weeks of assigned protocol both the groups were analyzed by VAS and Modified Oxford knee score.

\section{Results}

34 patients underwent Total Knee Replacement / Unicondylar Knee Replacement surgeries were screened for eligibility. 17 patients were randomized into control Group 1 (forward walking) while remaining 17 patients were randomized into experimental Group 2 (backward walking). Pain intensity was measured using VAS (Visual Analogue Scale). Disability and activity levels were measured using Modified Oxford knee score.

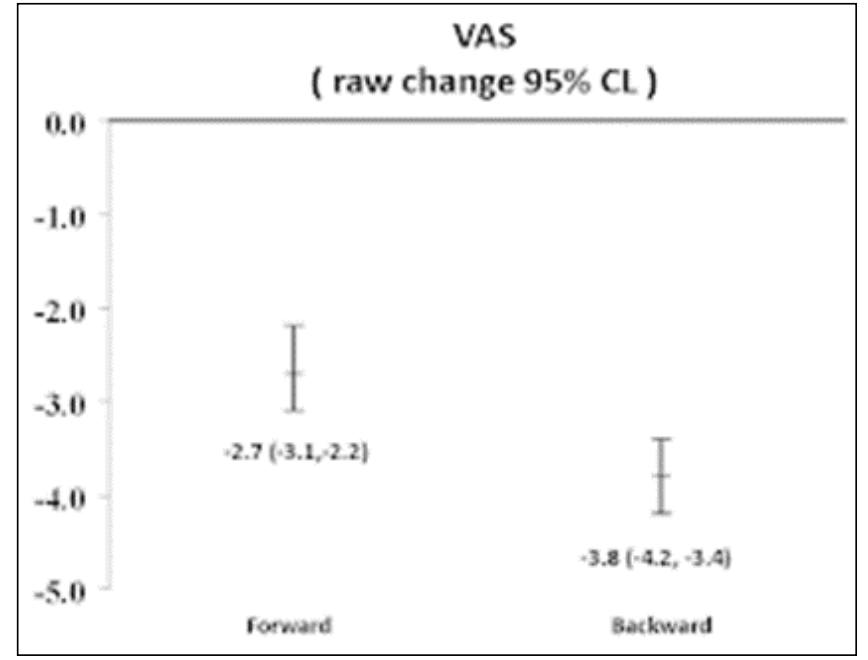

Chart 1: VAS score comparison between forward and backward walk (raw change)

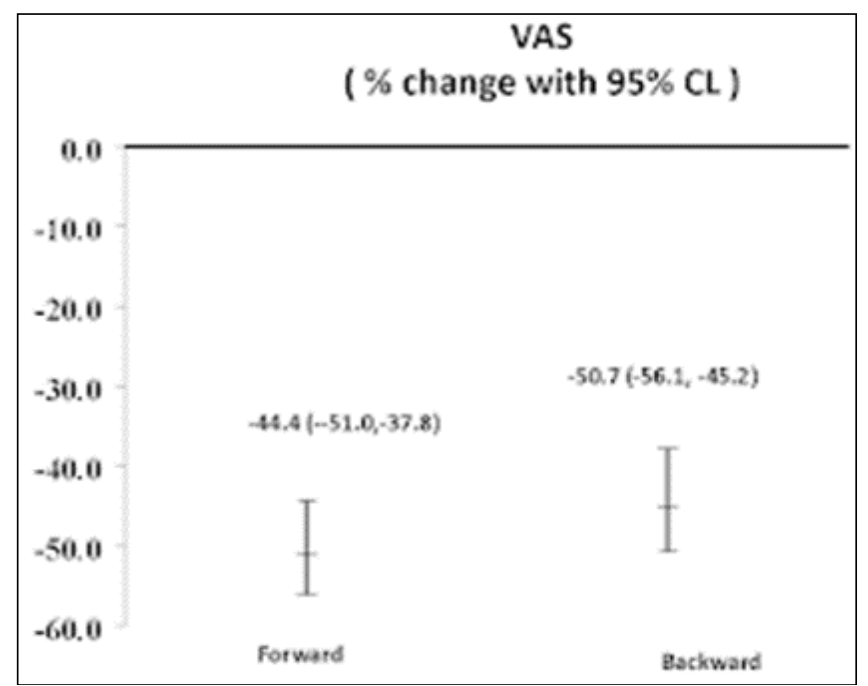

Chart 2: VAS score comparison between forward and backward walk (percentage Change)

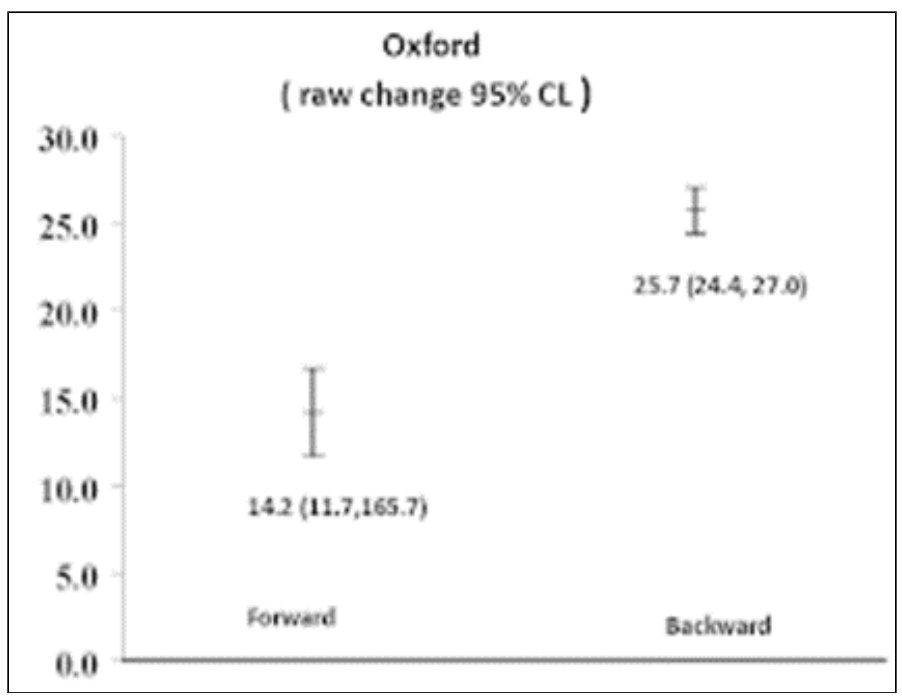

Chart 3: Modified Oxford Knee score comparison between forward and backward walk (raw change) 


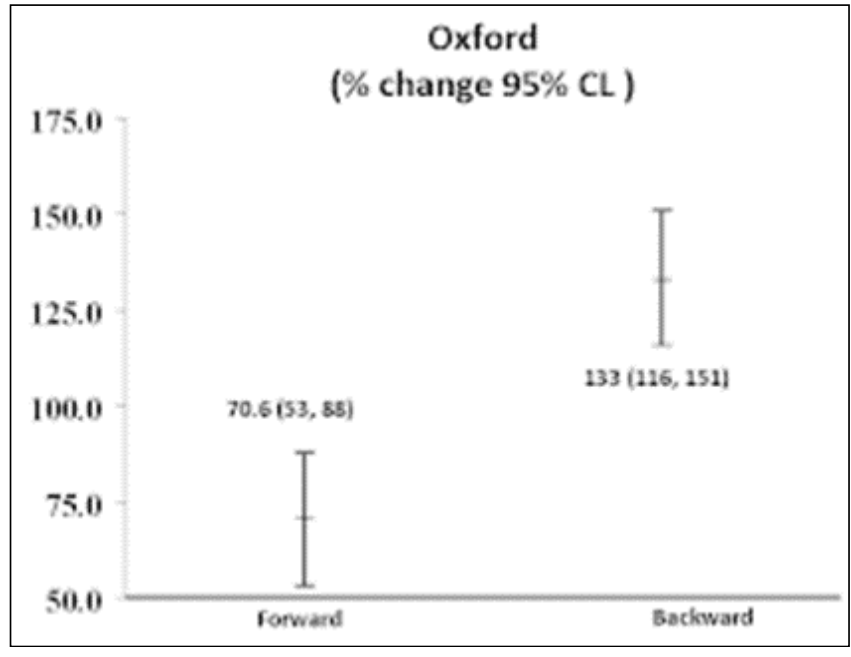

Chart 4: Modified Oxford Knee score comparison between forward and backward walk (percentage change)

Data was analyzed using SPSS 20.0. The results have been expressed as mean, standard deviation (SD) and $95 \%$ Confidence Interval (CI) p value of $<0.05$ was considered as statistically significant.

In group 1 there is significant reduction in pain intensity by VAS score. Improvement in VAS score of post-operative patient in group 1 is $44.4 \%$ (CL 51.0, 37.8). Improvement in VAS score of post-operative patient in group 2 is $50.7 \%$ (CL $56.1,45.2)$. There is additional improvement in VAS score in group 2.

Group 1 there is $70.6 \%$ (CL 53, 88) increase in post-operative Modified Oxford score. Group 2 there is significant $133 \%$ $(116,151)$ increase in post-operative Modified Oxford score, suggestive of additional $64.4 \%$ improvement in group 2 .

\section{Discussion}

Osteoarthritis knee is a metabolically active disease that includes both destruction and repair mechanism, destruction of cartilage and formation of osteophytes. Mechanical factors such as dynamic joint loading have been implicated in Osteoarthritis knee pathomechanics. Gait compensations secondary to joint stiffness and pain lead to greater imbalances of muscle, reducing endurance and flexibility and later ending in deformity. Patello-femoral loading mostly occurring in flexion is responsible for Patello-femoral arthritis. Hence the vicious cycle of Pain --> Fixed flexion deformity --> more patella-femoral degeneration --> more pain --> more Fixed Flexion Deformity etc.

Surgeries like Unicondylar knee replacement and Total knee replacement correct the morbid bony pathology. Physiotherapy in post-operative period is needed to correct the soft tissue dynamics and overall knee kinematic. Flexed knee causes more wear and tear and causes more fatigue.

Reverse walking is associated with fitness training, martial arts, mindfulness training. We decided to investigate whether it would help better and faster patient recovery post knee replacement surgery. It mainly acts through stretching posterior capsule, increasing quadriceps loading and decreasing Patello-femoral loading. Also it is associated with better proprioception.

Reverse walking puts less strain and requires less range of motion at knee joints. Also, because backward walking eliminates the typical heel-strike to the ground (the toe contacts the ground first), it can lead to changes in pelvis alignment that help open up the facet joints in your spine, potentially alleviating pressure that may cause low back pain in some people. ${ }^{[11,12]}$

Studies by Barry Bates and other authors have shown backward walking to have more advantageous effects than forward walking. During forward walking knee joint flexes, extends and then flexes in support phase, where as in backward walking knee initially extends, flexes and then extends in support phase. Hence there are 2 stages of extension in reverse walking vis-a-vis forward walking. ${ }^{[14,15]}$ Load on the knee is different due to first toe strike followed by heel strike \& weight bearing, compared to first heel strike followed by toe off in forward walking ${ }^{[16]}$.

This could also explain the higher activation of the knee extensors that help reduce fixed flexion contractures and give full expression to regaining the power of quadriceps.

Spine extensors are activated, Posture is more erect \& sagittal balance is healthier by avoiding the forward stooped posture. Studies by Flynn et al have shown backward walking produces significantly lower patellar compressive forces than forward walking ${ }^{[17,18]}$.

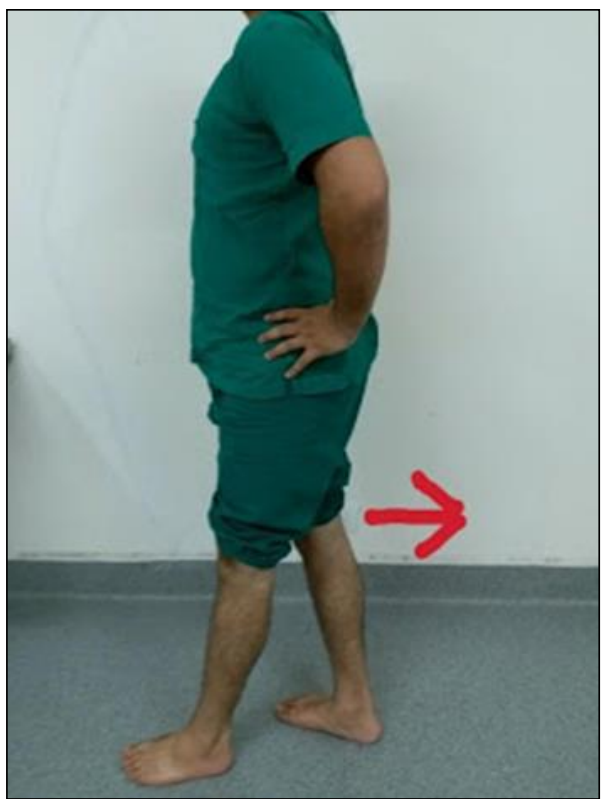

Image 1: Backward walking

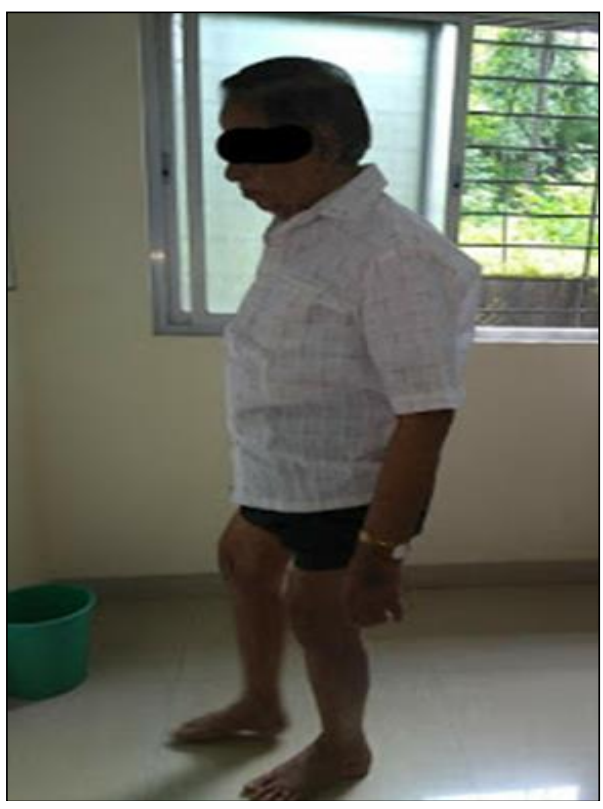

Image 2: forward walking 


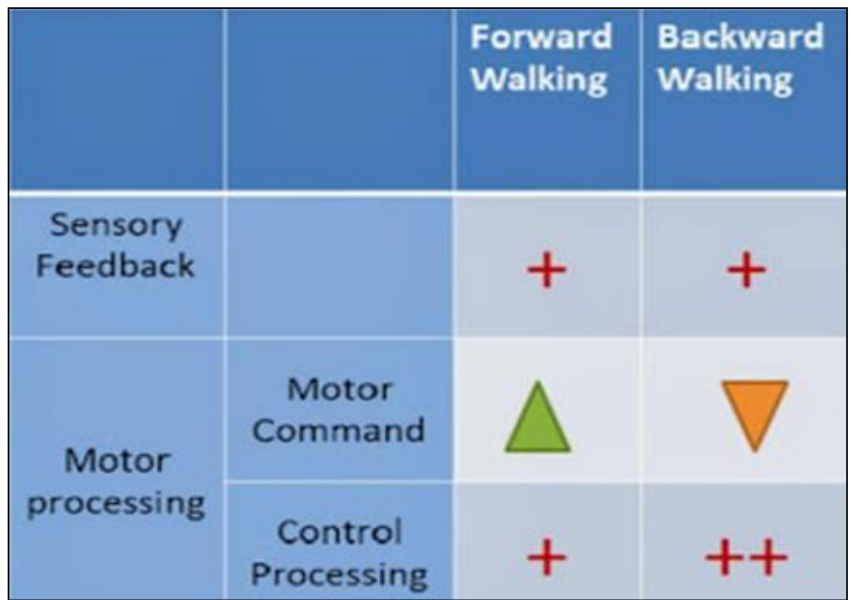

Image 3: Neurological processing difference

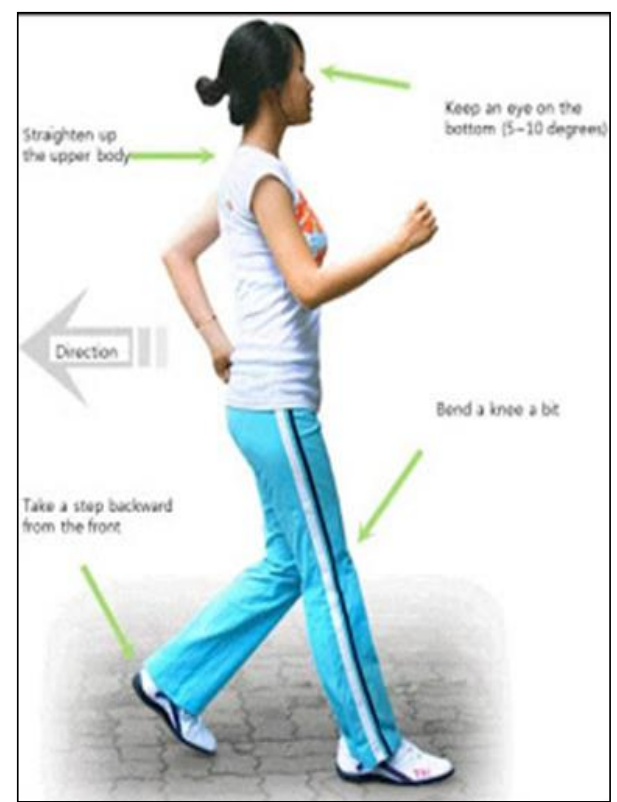

Image 4: backward walking technique

\section{Conclusion}

The above results conclude that backward walking can be especially advantageous for early rehabilitation of knee joint surgeries.

Among benefits of retro- walking is the balance gain related to forced reliance on the prospective inputs .Rear facing movements does not allow vision to orient to environmental objects to secure balance. The vestibular system is similar to other systems that depreciate if not worked reverse walking accomplishes this goal of stimulating balance by forcing a reliance on the vestibular system. Also our senses automatically build a defence mechanism against potential dangers. It gradually improves balance peripheral vision and hearing skills. Hence reverse walking should be included as a principle part of early protocols in post op rehabilitation for knee replacement surgeries.

\section{References}

1. Antony P, Leo Aseer1 G, Arun Maiya M, Mohan Kumar, Vijayaraghavan PV, content validation of total knee replacement rehabilitation protocol in Indian population: Journal of Clinical and Diagnostic Research. 2017; 11(6):YC05-YC09

2. Neil Artz, Karen $T$ Elvers, Catherine Minns Lowe, CathSackley, Paul Jepson, Andrew D Beswick.
Effectiveness of physiotherapy exercise following total knee replacement: systematic review and meta-analysis Artz et al. BMC Musculoskeletal Disorders. 2015; 16:15. DOI 10.1186/s12891-015-0469-6

3. Felson DT, Zhang Y. An update on the epidemiology of Hip and knee OA with view to prevention of Rheumatoid arthritis. 1998; 41:1343-1345

4. Kaufman KR, Hughes C, Morrey B. Gait characteristic of points with knee OA. Journal of biomechanics. 2001; 34:907-915

5. Khatri SM. Basics of orthopaedics PT. 6, 119-126

6. Marlon JM, Harmer A, Fransen M. Status of patients rehab following knee replacement in Australia. Physiotherapy Res. International. 2006; 11:35-47

7. RathiPalekar Varghese. Efficacy of backward walking on patients with OA knee on quads strength, pain and physical functions, Indian Journal of Physiotherapy and occupational therapy. 2014; 8:192-196

8. GauriGondhalekar, Medha Deo. Retrowalking as an adjunct to conventional therapy versus conventional therapy alone on painand disability with acute exacerbation of chronic OA knee. NAJMS. 5(2), 108-112

9. Campbell WI, Lewis S. Visual analogue measurement of pain. Ulster Med J. 1990; 59(2):149-154

10. Assessment of pain: H. Breivik1 2*, P. C. Borchgrevink4, S. M. Allen2, L. A. Rosseland2, L. Romundstad2, E. K. Breivik Hals3, G. Kvarstein2 and A. Stubhaug1 2 Published in British Journal of Anaesthesia. 2008; 101(1):17-24. doi:10.1093/bja/aen103 Advance Access publication May 16, 2008

11. https://fitness.mercola.com/sites/fitness/archive/2012/12/ 14/walking-backward.aspx

12. https://www.chatelaine.com/health/fitness/benefits-ofrunning-backwards/

13. Terblanche E, Page C, Kroff J, Venter RE. The effect of backward locomotion training on body composition and cardiorespiratory fitness of young women. International Journal of Sports Medicine. 2005; 26(3):214-219.

14. Winter DA, Pluck N, Yang JF, Journal of motor behaviour. Taylor \& Francis. Backward walking a simple reversal of forward walking, 1989.

15. Minhyeon Lee, Jungyoon Kim, Jonsang Son, Youngho Kim. Gait Posture. Kinematic and kinetic analysis during forward and backward walking. 2013; 38(4):674-678.

16. Vilensky JA, Gankie Wicz, Gehlsen G. A Kinematic comparison of Backward and forward walking in humans. Journal of human movement studies. 1987; $13: 29-50$

17. Flynn TW, Connery SM, Smutok MA, Zeballos RJ, Weisman IM. Comparison of cardiopulmonary responses to forward and backward walking and running. Medicine and Science in Sports and Exercise. 1994; 26:89-94

18. Flynn TW, Soutas-Little RW. Patellofemoral joint compressive forces in forward and backward running. Journal of Orthopedics and Sports Physical Therapy. $1995 ; 21: 277-282$.

19. https://wooridulhospital.wordpress.com/2016/03/07/walk ing-backward/ 